МИНИСТЕРСТВО ОБРАЗОВАНИЯ И НАУКИ РОССИЙСКОЙ ФЕДЕРАЦИИ

ТОМСКИЙ ГОСУДАРСТВЕННЫЙ УНИВЕРСИТЕТ

\title{
МАТЕРИАЛЫ
}

VIII Международной молодежной научной конференции

«МАТЕМАТИЧЕСКОЕ

И ПРОГРАММНОЕ ОБЕСПЕЧЕНИЕ

ИНФОРМАЦИОННЫХ, ТЕХНИЧЕСКИХ

И ЭКОНОМИЧЕСКИХ СИСТЕМ»

Томск, 26-30 мая 2021 г.

Под общей редакиией И.С. Шмырина

Томск

Издательство Томского государственного университета 2021 


\title{
VI. ТЕОРИЯ МАССОВОГО ОБСЛУЖИВАНИЯ И ЕЕ ПРИЛОЖЕНИЯ
}

DOI: 10.17223/978-5-907442-42-9-2021-21

\section{ИССЛЕДОВАНИЕ ХАРАКТЕРИСТИК РАБОТЫ ГИБРИДНОЙ ДВУХУРОВНЕВОЙ МОДЕЛИ ОБРАБОТКИ ЗАПРОСОВ}

\author{
Воркожоков А.А., Лапатин И.Л. \\ Томский государственный университет \\ vorkozhokovandrey@gmail.com, ilapatin@mail.ru
}

\section{Введение}

Одним из вариантов систем массового обслуживания являются многоуровневые системы, состоящие из нескольких СМО. В частности, двухуровневые системы с повторными вызовами были впервые рассмотрены C.M. Krishna и Y.H.Lee [1].

Системы массового обслуживания с повторными вызовами [2,3] представляют собой математические модели телекоммуникационных систем: компьютерных и телефонных сетей, систем передачи данных, радио, телевидения, мобильной связи и других. В нашем случае заявки обслуживаются по алгоритму CSMA (Carrier-sense multiple access), который используется для обмена данными в компьютерных сетях. Когда станция готова к передаче данных, она прослушивает канал. Если канал оказывается свободным, происходит передача кадра. Если же канал занят, то станция ждет в течение случайного интервала времени, а затем прослушивает линию снова. Наш входящий поток представляет собой суммарный поток всех обращений к каналу. При этом мы рассматриваем дополнительную фазу, которая может быть интерпретирована как фаза форматирования пакета для передачи по каналу. Данная фаза является некоторым фильтром, который контролирует количество запросов, поступающих в канал. Поэтому правильное построение фазы фильтрации позволит наиболее эффективно использовать пропускную способность канала. Мы будем исследовать количество обслуженных запросов узлом связи, т.е. выходящий поток нашего узла. Исследование характеристик выходящего потока при условии большой задержки на орбите [4] необходимо для исследования сетевых структур, в которых выходящий поток одного узла является входящим для другого.

\section{1. Описание математической модели}

В данной работе исследуется двухуровневая система массового обслуживания, которая на первом уровне содержит конечную очередь и неограниченную орбиту на втором уровне.

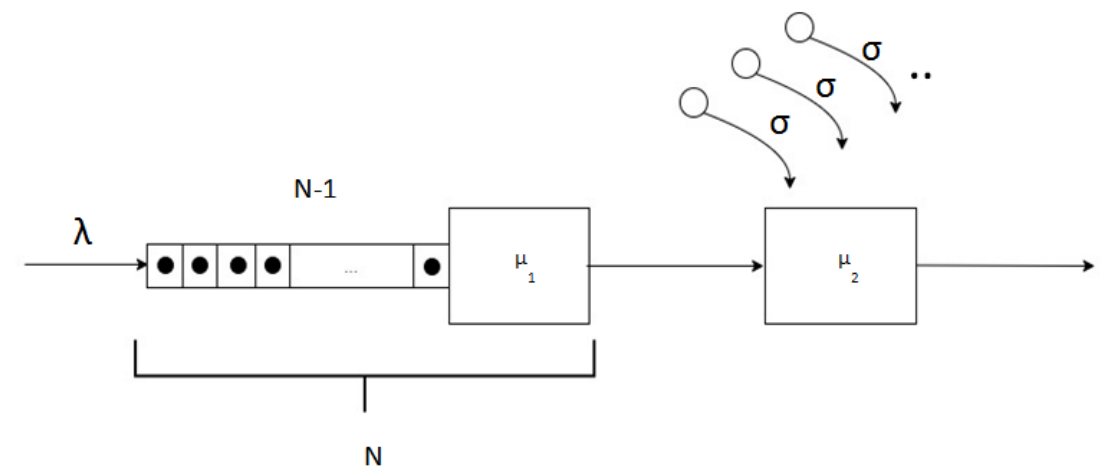

Рис. 1. Модель системы 
На вход первого уровня приходит простейший поток с параметром $\lambda$. Заявка начинает обслуживаться в течение времени, распределённого экспоненциально с параметром $\mu_{1}$, если в очереди перед ней нет заявок и прибор свободен. Если прибор занят и очередь не заполнена, то заявка встаёт в конец очереди. Если на первой фазе уже находится $N$ заявок, то вновь пришедшая заявка теряется.

После обслуживания на первой фазе на вход второго уровня приходит поток заявок. Поступая на вторую фазу и обнаруживая прибор свободным, заявка занимает его, а прибор начинает обслуживание в течение времени, распределённого экспоненциально с параметром $\mu_{2}$. Если же заявка, поступая в систему, обнаруживает прибор занятым, она мгновенно уходит на орбиту и осуществляет там случайную задержку в течение экспоненциально распределённого времени с параметром $\sigma$.

После обслуживания на двух уровнях заявка попадет в выходящий поток. Исследование характеристик именно выходящего потока является особенностью данной работы.

Опишем систему с помощью случайных процессов: $k(t)$ - число заявок на первой фазе в момент времени $t, i(t)$ - число заявок на орбите в момент времени $t, l(t)$ - состояния прибора на второй фазе в момент времени $t$ (0 - прибор свободен, 1 - прибор занят), $m(t)$ - число обслуженных заявок в момент времени $t$.

Теперь запишем распределение вероятностей для многомерного случайного процесса:

$$
P\{k(t)=k, i(t)=i, l(t)=l, m(t)=m\}=P_{l}(k, i, m, t) .
$$

Составим равенства, используя теорему о полной вероятности:

$$
\begin{gathered}
P_{0}(k, i, m, t+\Delta t)=P_{0}(k, i, m, t)(1-\lambda \Delta t)\left(1-\mu_{1} \Delta t\right)(1-i \sigma \Delta t)+ \\
+P_{1}(k, i, m-1, t) \mu_{2} \Delta t+P_{0}(k-1, i, m, t) \lambda \Delta t+o(\Delta t), \\
P_{1}(k, i, m, t+\Delta t)=-P_{1}(k, i, m, t)\left(\lambda+\mu_{1}+\mu_{2}\right)(1-\lambda \Delta t)\left(1-\mu_{1} \Delta t\right)\left(1-\mu_{2} \Delta t\right)+ \\
+P_{0}(k+1, i, m, t) \mu_{1} \Delta t+P_{1}(k+1, i-1, m, t) \mu_{1} \Delta t+P_{0}(k, i+1, m, t)(i+1) \sigma \Delta t+ \\
+P_{1}(k-1, i, m, t) \lambda \Delta t+o(\Delta t) .
\end{gathered}
$$

Далее, для того, чтобы описать переходы системы из одного состояния в другое, нам необходимо составить систему дифференциальных уравнений Колмогорова. Чтобы это сделать нужно в системе (1) представить $\Delta t \rightarrow 0$.

Составим систему дифференциальных уравнений Колмогорова:

$$
\begin{aligned}
& \frac{\partial P_{0}(k, i, m, t)}{\partial t}=-P_{0}(k, i, m, t)\left(\lambda+\mu_{1}+i \sigma\right)+P_{1}(k, i, m-1, t) \mu_{2}+P_{0}(k-1, i, m, t) \lambda, \\
& \frac{\partial P_{1}(k, i, m, t)}{\partial t}=-P_{1}(k, i, m, t)\left(\lambda+\mu_{1}+\mu_{2}\right)+P_{0}(k+1, i, m, t) \mu_{1}+ \\
& +P_{1}(k+1, i-1, m, t) \mu_{1}+P_{0}(k, i+1, m, t)(i+1) \sigma+P_{1}(k-1, i, m, t) \lambda . \\
& \text { Введём частичные характеристические функции: } \\
& H_{l}\left(k, u_{1}, u_{2}, t\right)=\sum_{i=0}^{\infty} \sum_{m=0}^{\infty} e^{j u_{1} i} \cdot e^{j u_{2} m} \cdot P_{l}(k, i, m, t) \text {. Подставим их в систему уравнений (2): } \\
& \frac{\partial H_{0}\left(k, u_{1}, u_{2}, t\right)}{\partial t}=\frac{\partial H_{0}\left(k, u_{1}, u_{2}, t\right)}{\partial u_{1}} j \sigma-H_{0}\left(k, u_{1}, u_{2}, t\right) \lambda-H_{0}\left(k, u_{1}, u_{2}, t\right) \mu_{1}+ \\
& +H_{1}\left(k, u_{1}, u_{2}, t\right) \mu_{2} e^{j u_{2}}+H_{0}\left(k-1, u_{1}, u_{2}, t\right) \lambda, \\
& \frac{\partial H_{1}\left(k, u_{1}, u_{2}, t\right)}{\partial t}=-H_{1}\left(k, u_{1}, u_{2}, t\right)\left(\lambda+\mu_{1}+\mu_{2}\right)+H_{0}\left(k+1, u_{1}, u_{2}, t\right) \mu_{1}+ \\
& +H_{1}\left(k+1, u_{1}, u_{2}, t\right) \mu_{1} e^{j u_{1}}-\frac{\partial H_{0}\left(k, u_{1}, u_{2}, t\right)}{\partial u_{1}} j \sigma e^{-j u_{1}}+H_{1}\left(k-1, u_{1}, u_{2}, t\right) \lambda .
\end{aligned}
$$


Теперь представим систему в матрично-векторном виде. Так как процесс $k(t)$ ограничен, мы можем утверждать, что параметр $k$ принимает значения от 0 до $N$. Введём вектор характеристических функций размерностью $N+1$, где вместо параметра $k$ запишем его значения:

$$
\mathbf{H}_{l}\left(u_{1}, u_{2}, t\right)=\left\{H_{l}\left(0, u_{1}, u_{2}, t\right), H_{l}\left(1, u_{1}, u_{2}, t\right), \ldots, H_{l}\left(N, u_{1}, u_{2}, t\right)\right\},
$$

и матрицы размерностью $(N+1) \times(N+1)$ :

$$
\mathbf{B}=\left(\begin{array}{ccccc}
0 & 0 & \ldots & 0 & 0 \\
1 & 0 & \ldots & 0 & 0 \\
\ldots & \ldots & \ldots & \ldots & \ldots \\
0 & 0 & \ldots & 0 & 0 \\
0 & 0 & \ldots & 1 & 0
\end{array}\right), \mathbf{B}^{T}=\left(\begin{array}{ccccc}
0 & 1 & \ldots & 0 & 0 \\
0 & 0 & \ldots & 0 & 0 \\
\ldots & \ldots & \ldots & \ldots & \ldots \\
0 & 0 & \ldots & 0 & 1 \\
0 & 0 & \ldots & 0 & 0
\end{array}\right), \mathbf{A}=\left(\begin{array}{ccccc}
\lambda & 0 & \ldots & 0 & 0 \\
0 & \lambda+\mu_{1} & \ldots & 0 & 0 \\
\ldots & \ldots & \ldots & \ldots & \ldots \\
0 & 0 & \ldots & \lambda+\mu_{1} & 0 \\
0 & 0 & \ldots & 0 & \mu_{1}
\end{array}\right) .(5)
$$

Подставим (4) и (5) в систему (3):

$$
\begin{gathered}
\frac{\partial \mathbf{H}_{0}\left(u_{1}, u_{2}, t\right)}{\partial t}=\frac{\partial \mathbf{H}_{0}\left(u_{1}, u_{2}, t\right)}{\partial u_{1}} j \sigma-\mathbf{H}_{0}\left(u_{1}, u_{2}, t\right)\left[\lambda \mathbf{B}^{T}-\mathbf{A}\right]+\mathbf{H}_{1}\left(u_{1}, u_{2}, t\right) \mu_{2} e^{j u_{2}}, \\
\frac{\partial \mathbf{H}_{1}\left(u_{1}, u_{2}, t\right)}{\partial t}=\mathbf{H}_{1}\left(u_{1}, u_{2}, t\right)\left[\lambda \mathbf{B}^{T}-\mathbf{A}-\mu_{2} \mathbf{I}+\mu_{1} e^{j u_{1}} \mathbf{B}\right]+ \\
+\mu_{1} \mathbf{H}_{0}\left(u_{1}, u_{2}, t\right) \mathbf{B}-\frac{\partial \mathbf{H}_{0}\left(u_{1}, u_{2}, t\right)}{\partial u_{1}} j \sigma e^{-j u_{1}} .
\end{gathered}
$$

\section{2. Асимптотический анализ}

Для исследования характеристик выходящего потока, возьмём условие большого времени задержки заявки на орбите.

Сделаем замену:

$$
\sigma=\varepsilon, u_{1}=\varepsilon w, \mathbf{H}_{l}\left(u_{1}, u_{2}, t\right)=\mathbf{F}_{l}\left(w, u_{2}, t, \varepsilon\right) .
$$

Подставим (7) в систему (6):

$$
\begin{gathered}
\frac{\partial \mathbf{F}_{0}\left(w, u_{2}, t, \varepsilon\right)}{\partial t}=\frac{\partial \mathbf{F}_{0}\left(w, u_{2}, t, \varepsilon\right)}{\partial w} j-\mathbf{F}_{0}\left(w, u_{2}, t, \varepsilon\right)\left[\lambda \mathbf{B}^{T}-\mathbf{A}\right]+\mathbf{F}_{1}\left(w, u_{2}, t, \varepsilon\right) \mu_{2} e^{j u_{2}}, \\
\frac{\partial \mathbf{F}_{1}\left(w, u_{2}, t, \varepsilon\right)}{\partial t}=\mathbf{F}_{1}\left(w, u_{2}, t, \varepsilon\right)\left[\lambda \mathbf{B}^{T}-\mathbf{A}-\mu_{2} \mathbf{E}+\mu_{1} e^{j \varepsilon w} \mathbf{B}\right]+ \\
+\mu_{1} \mathbf{F}_{0}\left(w, u_{2}, t, \varepsilon\right) \mathbf{B}-\frac{\partial \mathbf{F}_{0}\left(w, u_{2}, t, \varepsilon\right)}{\partial w} j e^{j \varepsilon w} .
\end{gathered}
$$

Теорема. Асимптотическое приближение двумерной характеристической функции числа обслуженных заявок входящего потока за некоторое время $t$ имеет вид

$$
\begin{gathered}
\lim _{\sigma \rightarrow 0} \mathrm{M} e^{j u_{2} m}=\lim _{\varepsilon \rightarrow 0} \sum_{l=0}^{1} \mathbf{H}_{l}\left(0, u_{2}, t\right) \mathbf{E}=\lim _{\varepsilon \rightarrow 0} \sum_{l=0}^{1} \mathbf{F}_{l}\left(0, u_{2}, t, \varepsilon\right) \mathbf{E}= \\
=\sum_{l=0}^{1} \mathbf{F}_{l}\left(u_{2}, t\right) \mathbf{E}=\mathbf{R} e^{\mathbf{J}\left(u_{2}\right) t} \mathbf{E} \mathbf{E},
\end{gathered}
$$

где $\mathbf{R}=\left\{\mathbf{R}_{1}, \mathbf{R}_{2}\right\}-$ стационарное распределение вероятностей, $\kappa-$ среднее число заявок на орбите [5], $\mathbf{E}$ - вектор размерностью $N+1, \mathbf{E E}-$ вектор размерностью $(2 N+2) \times(2 N+2), \mathbf{J}\left(u_{2}\right)=\left[\begin{array}{cc}\kappa \mathbf{I}-\lambda \mathbf{B}^{T}+\mathbf{A} & \mu_{1} \mathbf{B}-\kappa \mathbf{I} \\ \mu_{2} e^{j u_{2}} \mathbf{I} & \lambda \mathbf{B}^{T}-\mathbf{A}-\mu_{2} \mathbf{I}+\mu_{1} \mathbf{B}\end{array}\right]$. 
Чтобы перейти от характеристических функций обратно к распределению, нам необходимо воспользоваться обратным преобразованием Фурье:

$$
P\{m(t)=m\}=P(m, t)=\frac{1}{2 \pi} \int_{-\pi}^{\pi} e^{-j u_{2} m} \mathbf{R} e^{\mathbf{J}\left(u_{2}\right) t} \mathbf{E} \mathbf{E} d u_{2} .
$$

\title{
Заключение
}

В ходе работы была рассмотрена гибридная система с очередью на первой фазе и орбитой на второй. Для описания модели была составлена система дифференциальных уравнений Колмогорова (2). С использованием метода частичных характеристических функций и метода асимптотического анализа была получена формула (8), являющаяся асимптотическим приближением характеристической функции числа событий, наступивших в выходящем потоке. С применением к полученной формуле (8) обратного преобразования Фурье было получено распределение вероятностей числа событий, наступивших в выходящем потоке за время $t(9)$.

\section{ЛИТЕРАТУРА}

1. Krishna C.M., Lee Y.H. A study of two-phase service // Operations Research Letters. - 1990. - V. 9. - P. 91-97.

2. Kendall D.G. Stochastic processes occurring in the theory of queues and their analysis by the method of the imbedded Markov chain. - The Annals of Mathematical Statistics, 1953. - P. 338-354. $309 \mathrm{p}$

3. Artalejo J.R., Gomez-Corral A. Retrial Queueing Systems: A Computational Approach. - Springer, 2008. -

4. Nawel Gharbi, Claude Dutheillet An algorithmic approach for analysis of finite-source retrial systems with unreliable servers // Computers \& Mathematics with Applications. - 2011. - V. 62, Iss. 6. - P. 2535-2546.

5. Назаров А.А., Анисимова А.А. Асимптотический анализ первого порядка двухфазной СМО с конечной очередью и орбитой в условии большой задержки заявок на орбите.// Молодежная научная школа по прикладной теории вероятностей и телекоммуникационным технологиям (АРТСТ-2017): материалы молодежной научной школы. Россия, Москва, 23-27 октября 2017 г.; под общ. ред. К.Е. Самуйлова, Е.А. Кучерявого, А.Н. Дудина. Москва : РУДН, 2017. - С. 175-177.

DOI: $10.17223 / 978-5-907442-42-9-2021-22$

\section{ИССЛЕДОВАНИЕ НАДЕЖНОСТИ ДУБЛИРОВАННОЙ СИСТЕМЫ \\ С ПОЛНЫМ ВОССТАНОВЛЕНИЕМ ПРИ ПРОИЗВОЛЬНЫХ ДЛИТЕЛЬНОСТЯХ ЖИЗНИ И РЕМОНТА ЕЁ ЭЛЕМЕНТОВ}

\author{
Иванова Н.М. ${ }^{1,2}$, Нибасумба Э. ${ }^{1}$ \\ ${ }^{1}$ Российский университет дружбы народов \\ ${ }^{2}$ Институт проблем управления им. В.А. Трапезникова РАН \\ nm_ivanova@bk.ru, ema.patiri2015@yandex.ru
}

\section{Введение}

Теория регенерирующих процессов была предложена Смитом в 1955 г. [1] в качестве нового метода исследования случайных процессов. Эта теория нашла множество обобщений (см., например, [2,3]) ввиду широкого круга применений к прикладным задачам. Исследование систем с произвольными распределениями времен жизни и ремонта их компонент важно как с теоретической точки зрения, так и прикладной. В [4] для функций распределения длительностей безотказной работы и ремонта были введены модифицированные преобразования Лапласа-Стилтьеса. На основе этого преобразования в [5] с помощью теории разложимых полуругеренерирующих процессов [6] были найдены аналитические выражения для основных характеристик надежности данной системы при сценарии частичного ремонта. Настоящая работа продолжает ис-

" Публикация выполнена при поддержке Программы стратегического академического лидерства РУдН и при финансовой поддержке РФФИ в рамках научного проекта № 20-01-00575А (Иванова Н.М., проведение аналитических расчетов). 